\title{
Accelerating Decision-Making in Transport Emergency with Artificial Intelligence
}

\author{
Alexander Raikov* \\ Institute of Control Sciences, Russian Academy of Sciences, 119526, Russia
}

\begin{tabular}{l} 
A R T I C L E I N F O \\
\hline Article history: \\
Received: 04 March, 2020 Accepted: 10 July, 202 \\
Online: 20 November, \\
\hline Keywords: \\
Artificial intelligence \\
Big data analyses \\
Cognitive modeling \\
Convergent approach \\
Virtual meetings \\
Transport emergency
\end{tabular}

\section{Introduction}

Every second has great importance after the moment of the outbreak of transport emergencies. The first hour after the moment is named the "golden hour"-it is a critical time for saving the injured and suffered lives. Different types of transport entities may take part in automobile, railway, aircraft, all-terrain vehicle (ATV), motorcycle, bicycle, etc. The authorities and their departments, rescue teams, police, ambulance staff, representatives of the population, etc. may participate in the processes. They all must understand each other and get agreement on decisions very quickly.

Rescue teams usually have a special pre-designed guide to synchronize their participants' activities as usual. It may be represented by way of detailed prewritten instructions, plans, or models of activity. The members of the rescue team must know it in advance. But every emergency is characterized by its own

\footnotetext{
*Corresponding Author: Alexander Raikov, alexander.n.raikov@gmail.com
}

features and new participants, and the standard guide sometimes can only confuse the situation. The activity plans of all participants must be synchronized in any circumstances and for different environments. The integrity of the team member tactics' implementations has to achieve the proper synergistic effect.

Special decision support systems have been created based on virtual reality tools and artificial intelligence (AI) technologies. A sharp rise of research and the creation of such systems for this activity began after the 9/11 attacks and the 2009 crash of an Air France Airbus A330. The situational awareness (SA) approach [1, 2] was suggested and used in researching these disasters. Profound technical decisions were found, and obvious progress was made [3].

Simultaneously, the crucial importance of accelerating the collective decision-making by getting agreement between different participants in extreme conditions makes the issue of speeding up virtual meetings in emergencies always relevant. Some author's 
papers are devoted to accelerating distributed collective decisionmaking that can be held in situational centers (SC) or a networked environment [4]. It was already suggested the technologies that help: speeding up a corporate strategic conversation [4], raising the quality of networked experts' procedures [5], improving medicine meeting (conversation) [6], or even accelerating networked democracy processes [7] and using for these methods from quantum theory $[8,9]$.

Brainstorming procedures are divergent as usual; they have goals of creating a lot of new ideas. But these ideas may not be consistent, and as a result, synergy cannot be achieved. The author's convergent approach ensures the purposefulness and sustainability of networked collective meetings $[4,5]$. It has been tested in different fields of the social-economy sphere and for decision-making in SC. It is now suggested for networked and collective decision-making support in emergencies when many different participants and physical automated tools (computers, cars, and intellectual robots) take part.

Three meeting modes may be distinguished: monitoring the situation with an operational solution of ongoing issues, planned decision-making with long-term preliminary preparation, and decision-making in an emergency. The latter is the most difficult. It claims a special approach that will ensure the convergence of distributed meetings and conversations to save the lives of as many injured people as possible. The paper has devoted this mode.

The paper includes a description of a transport emergency's characteristics, considers the limitations of the available rescue means of situational awareness. The concepts of denotative (formalized) semantics and cognitive (non-formalized) semantics for AI models are introduced, wherein, shows that modern AI predominantly uses only the former one. Further, the paper examines the possibilities of advanced AI and proposes to consider the possibility of using quantum theory to construct cognitive semantics. The author's convergent approach is then proposed to speed up networked distributed meetings in conditions of transport emergency. Simultaneously, the author's approach to the organization of networked expertise and cognitive modeling is considered in more detail. Then, some practical implementations of this approach are demonstrated. The paper ends with the formulation of discussion questions.

\section{Emergency Features}

The following features characterize an emergency transport situation (railway, aviation, highway, etc.):

- Presence of injured and/or dead.

- Victims need quick medical attention.

- Extreme lack of time.

- Possibility of destruction or contamination of the area.

- Participation of many departments, organizations, and rescue teams in decision-making.

- Distribution of collective decision-making process.

- Lack of material resources in the proper amount.

- Experienced experts are not available, etc.
In such situations, special tools have been created to speed up and improve the quality of collective decision-making, including virtual collaboration systems and SA systems [3]. They differ significantly from traditional teleconference. They are based on the advanced technology of discourse processing to ensure accelerated mutual understanding during participants' communications. SA is associated with multidimensional visualization, targeted information. It supports group decision-making, perception, prediction of the external environment changes, and mutual understanding between participants.

It is shown that in emergencies, network teams act faster than hierarchical ones $[1,5,7]$. The critical factors are as follow lack of necessary information, a holistic view of the situation at all levels of management, and the absence of an overall operational picture. Moreover, the growth of data volumes and the number of precedents do not always improve SA and quality decisions. The main sources of the problems that create information gaps are the team members themselves. They are focusing on solving their own problems. Inaccurate and belated information about events is the reason for information gaps too. The aspect of the lack of trust is important. Recently, the concept of SA is increasingly a group character: participants have to get an equally understanding of an operational picture when information is visualized on a map.

The analysis of the issue of accelerating the group decisionmaking in emergency using SA and virtual collaboration approaches showed the emphasis of the engineers' and researchers' efforts on the following areas:

- The creation of a distributed SC for monitoring and managing the processes of eliminating the consequences.

- Forecasting the danger of traffic accidents for ensuring effective decision-making.

- Integrating methods of information and analytical support crowdsourcing and networked expertise processes.

- Ensuring accelerated convergence of decision-making processes based on cognitive modeling and inverse problemsolving methods, etc.

One of the most efficient approaches to improve group decision-making processes in emergencies is SA. Let us take a look at it in more detailed.

\section{Situational Awareness}

The SA process embraces individuals, teams, AI systems, machines, and computer tools. It includes several stages $[1,3,10]$, has a gradation in levels [11], and is characterized by different models [12]. The process may have abnormal behavior that is defined as behavior to deviate from the global plan in some circumstances.

It is known that SA systems do not always behave sustainable and purposeful. Some studies try to represent cognitive nonformalized processes by linking participants with AI systems. It allows each actor to get information in special templates from other actors that help to increase the opportunities of all actors [13]. Nonpredicted and false responses are still common [3]. It is usually considered that SA is a suitable framework for collective cognitive 
modeling that helps to make effective decisions by using explicit and implicit information.

But most papers do not consider the chaotic nature of human cognition, especially in cases of responding to abnormal machine behavior. Participants' thoughts, emotions, free will, experience, senses, etc. cannot be described logically. It cannot be represented in computers' logical knowledge bases. As soon as an attempt to describe an emotion is made, this emotion disappears or loses its nature and creative power. This fact compels to consider simultaneously two types of cognitive processes - systems 1 and 2 as shown in Table 1.

Table 1: Two types of cognitive systems

\begin{tabular}{|c|l|l|l|}
\hline $\begin{array}{c}\text { System } \\
\text { number }\end{array}$ & Operating means & $\begin{array}{l}\text { Semantics } \\
\text { type }\end{array}$ & Serves to ensure \\
\hline 1 & $\begin{array}{l}\text { Non-logically, } \\
\text { unconsciously, } \\
\text { uncaused, } \\
\text { associatively, } \\
\text { intuitively }\end{array}$ & Cognitive & $\begin{array}{l}\text { Provides creativity, } \\
\text { helps to get } \\
\text { insights[8], } \\
\text { "Eureka effect" } \\
{[15]}\end{array}$ \\
\hline 2 & $\begin{array}{l}\text { Logically, } \\
\text { deliberately, } \\
\text { rationally and } \\
\text { analytically; neuron } \\
\text { deep learning } \\
\text { (partially) }\end{array}$ & Denotative & $\begin{array}{l}\text { Logical inference; } \\
\text { provides } \\
\text { necessary } \\
\text { conditions for } \\
\text { purposeful and } \\
\text { sustainable } \\
\text { decision-making } \\
\text { [4]. }\end{array}$ \\
\hline
\end{tabular}

The systems are interacting. Some researchers argue that the system 1 has an advantage over the second one. But it obvious that intuition is not only the ability to make a decision.

Relevant information of the system 2 may be claimed to avoid mistakes in judgment [14]. This system gives the possibility to make the features of human activity and task characteristics distinct. An example of the presentation of system 2 is big data. They are used to build denotative semantics. However, it should be noted that the juxtaposition of each factor or connection of the AIs' models with some relevant subset of big data will require the use of special methods for solving problems on cognitive models, namely, methods for solving large-scale global optimization problems [15]. But due to the very high requirements for the promptness of solving problems in emergencies, most likely, such an approach for such situations is unacceptable. This paper proposes replacing big data subsets with their projections on a deep neural network (See Section 8).

The creative process of getting an insight may be non-logical; the result appears after deliberate and sometimes long-time unsuccessful thinking. It needs to use logical efforts to speed up the process. [16]. It was suggested to put a cognitive continuum of the first above-mentioned process in the unconscious end of some scale, but the second one-in the conscious end [17]. Distinctions between these two processes have a continuum power. Effective decision-making may require flexibility on this continuum. And intuitive decision-making can be partly conscious, and analytical decisions can be partly unconscious.

Situational awareness in emergencies is working with machines and humans. It needs to control machines' abnormalities and supports correct group decision-making during abnormal machine behaviors. The three-level SA model was suggested for these cases [18]. It includes the following components:

- Perception of the situation (level 1).

- Comprehension of the situation's meaning and assessment relevance to certain goals (level 2).

- The projection of its status and dynamics for the near future (level 3).

Many relevant issues in different safety domains (aircraft, air traffic control, power plants, maritime operations, manufacturing systems, collaborative and isolated work, healthcare, etc.) benefit from the application of the three-level SA approach.

As has been shown, SA can cover a wide range of cognitive concepts. It is affected by psychological phenomena such as stress, mental workload, and trust [19]. The three-level SA approach was used in deliberate sense-making, story construction, recognizing main decisions, heuristics, and implicit cues of sociotechnical systems. The three-level SA approach has been used to explain deliberate attempts of detecting, diagnosing, and forecasting and increasing collective understanding of the problem situation.

An important aspect of SA is the sense-making process that explains how humans understand the environment by assigning meaning to experiences [20]. Sense-making is about values, priorities, and preferences. It holds different elements together to guide actions. Sense-making is a complex process and involves previously accumulated information. Different authors have tried to model cognition by identifying cues, diagnosing problems, taking action, questioning, elaborating, comparing, switching, and abandoning the search for a frame [21].

Distributed SA (DSA) is a more difficult construction than SA [22]. Its attention devotes to special issues of covering distributed cognition and focuses on collective SA, or awareness between agents. Humans and machines can represent them. DSA is used in modeling virtual collaborative processes.

DSA differs from SA substantially. For example, for DSA, it does not matter if the human agent does not know anything. Other components of the distributed system can augment the lack of his or her knowledge. On the contrary, in the case of SA, if the human does not know something, it may cause a significant problem or accident. But SA can support a broader spectrum of cognition because of the implicit character of thinking.

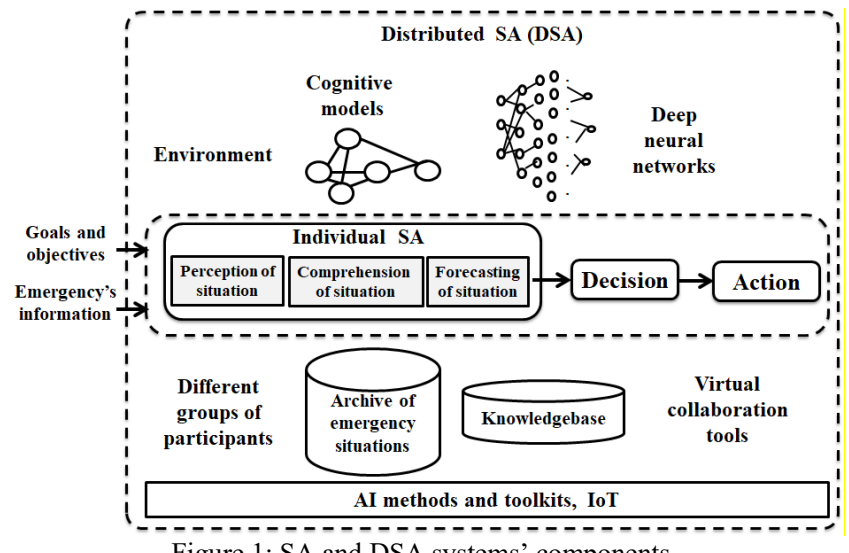

Figure 1: SA and DSA systems' components 
It was identified that there are four classes of failure transactions that can be the reason for the accident [3]: absent transactions, inappropriate transactions, incomplete transactions, and misunderstood transactions. Every one of these transactions has implicit and explicit interpretations, intangible and tangible representations. SA and DSA have framework constructions that can be represented in a formalized way by frames, ontology, or proposition logic. The components of SA and DSA systems are illustrated in Figure 1, where the idea of individual construction has been taken from [3].

Thus, SA and DSA have two semantics' interpretations: cognitive (non-formalized process or System 1) and denotative (formalized process or System 2).

\section{Cognitive and Denotative Semantics}

Emotions, thoughts, insights, intuition, inspiration, etc. come without awareness. They are usually defined as the capability to act or make decisions appropriately without consciously balancing alternatives, without following a certain rule.

Human and AI cognitive abilities are deep discussed in [23]. This paper analyzed 2500 publications. There are about three hundred cognitive architectures that describe human cognitive abilities. This paper's main attention was made to a logical description of cognitive phenomena. It was also noted that the topic of cognitive architectures is rather rare in works devoted to Artificial General Intelligence (AGI).

Therefore, SA or DSA does not cover this communication layer and participants' activities or covers it only in a very restricted means. They do not fully reflect the semantics generated by thoughts and feelings. In the SA or DSA paradigm, only formalized semantics can be represented. Moreover, in an emergency, technical devices and computers reflect only formalized, material aspects of the situation.

A machine has its own personality, which could be defined as only the technical perception of everything the participants know and feel [24]. Humans have the ability to apply their senses to identify and interpret signals to get their own useful descriptions of machine behavior. As a result, tacit knowledge [25] cannot be embraced by computer AI models; the computer cannot understand them; therefore, digital information from sensors may be estimated in the wrong way.

\section{Emergency's participants}

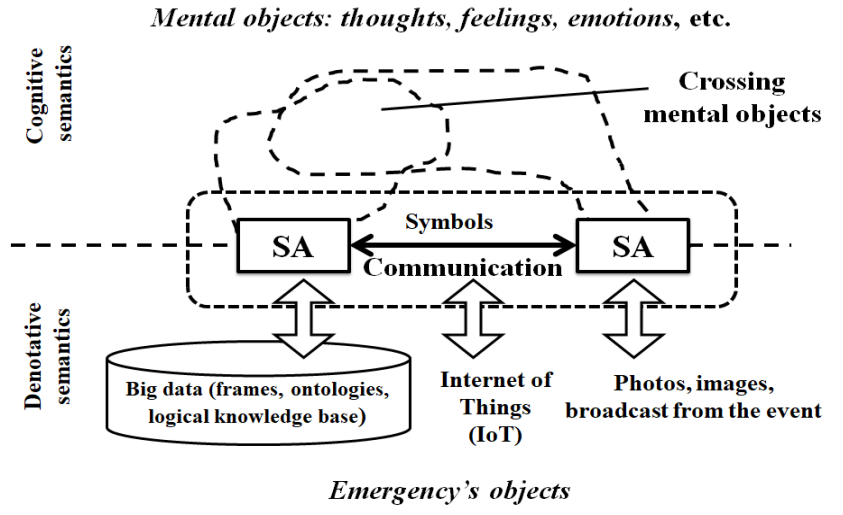

Figure 2: SA and its semantics: cognitive and denotative
Thus, there are two types of the semantics of SA or DSA: denotative (formalized) and cognitive (non-formalized)-see above Table. These semantics are illustrated in Figure 2.

Figure 2 demonstrates that the denotative semantics of SA and DSA has a formalized structure and can be implemented using logical ontologies, symbols, and frames. The instruments of the above-mentioned SC [4] can be useful for centralizing the control of monitoring problems' situations' and ensuring coherence of group decision-making and actions of various teams and organizations. SC helps make group decision-making processes stable (sustainable) and purposeful by involving AI and virtual collaboration technology to give participants the power to achieve synergy in the decision-making processes [26]. SC is using for solving problems that can be characterized by the following specific features:

- $\quad$ Ill-defined and weakly formalized problems.

- Require urgent collective decisions.

- Associated with high risks.

- Poorly predictable and chaotic behavior.

- The process can be interrupted.

- Divergent trait of breakthrough procedures.

- Lack of retrospective experience, etc.

SC usually exploits modern technologies by integrating:

- Strategic analysis methods;

- $\quad$ SA and DSA technologies.

- Advanced tools of visualization.

- Networked convergent conversation technology.

- Cognitive modeling.

- Inverse problem-solving methods.

- Logical analysis, statistical forecasting.

- $\quad$ Big data analysis, knowledge engineering.

- Different AI technologies, etc.

Simultaneously, the cognitive semantics cannot be fully taken into consideration by these technologies because of its nonformalized stuff. They have a more qualitative and non-metric nature than quantitative and metric ones. The cognitive aspects of decision-making and modeling in the SC have to ensure new requirements for cognitive semantic interpretations of SA or DSA. The main idea of making cognitive semantic interpretation by logical computer models consists of:

- Taking into consideration individual and collective unconscious phenomena using indirect and inverse methods of decision support.

- Impacting on the problem situation through a self-developing reflexive-active environment.

Using the ideas of AGI and Strong AI perhaps can help realize this idea. Strong AI and AGI are usually considered different 
phenomena [27], but in this paper, we will consider them synonyms without loss of generality. However, considering the practical need to introduce AI tools shortly, the main emphasis will be on using traditional AI, although more developed cognitive semantics are used in its application.

\section{Artificial General Intelligence}

The traditional AI has been developed on a logical basis. It uses predicate calculus, logical ontologies, frames, and neural networks $(\mathrm{NN})$. It may be noticed that the latter can be represented by logic also. This AI has a strong potential for solving different issues in real practice, such as predicting human behavior and different situations based on experience. This traditional approach can help to recognize the problem situation based on retrospective deep learning. Since traditional AI has a formalized nature, its semantics is denotative.

Currently, the traditional formalized AI gives way to nonformalized and uncaused AI, the semantics of which have cognitive features. The design of AGI can obey reverseengineering rules. Simultaneously, in the history of AI development, there are roughly four methodologies to achieve kinds of AI: symbolism, connectionism, behaviorism, and statisticalism [27].

AGI is usually considered the machine intelligence that can perform intellectual problems in different fields of knowledge more efficiently than a human being can adapt to the environment and forms its own self-awareness. It is fundamentally nonformalizable human-machine (hybrid) AI. The AGI encompass the subjective, mental, creative, and intellectual activity of human being. The distinctive claims of the AGI are as follows [28]:

- Creation of the necessary conditions (see Section 7) to ensure the convergence of generating ideas and thoughts to illdefined goals.

- Complex cognitive semantic interpretation of different problem situations' models.

- Taking into consideration the conceptual aspects of the situation.

- Ensure the sustainability and purposefulness of current and future development.

- Computers' models may be changed in a quantized way.

- The behavior of models is synchronized with the behavior of remote objects, the states of which are unknown.

- The space of modeling is conceptual, phenomenological, and uncaused.

- An external impact on the problem situation can be changed unpredictably, etc.

Thus, these claims have to be taken into account during organizing distributed meetings. It requires changing the methodological that was based on traditional AI. The possibilities of AGI's elements have to be taken into account. AGI includes all the advantages of $\mathrm{AI}$ and is intended to make a wide range of human cognitive capabilities. But for a while, it is obvious that there is a large gap between AI and AGI. It may be just said that
AGI is the inverse of AI [27,28]. In this context, it is necessary to consider such aspects of AGI as:

- Imitation of human consciousness, perhaps, with nonclassical methods from such disciplines, as relativistic and quantum physic, thermodynamics, and optics.

- Take into account cosmological, relativistic, atomic, quarks, and microtubule components of the brain.

- Take into account the physical fields (gravitational, electromagnetic, strong, and weak) on the thoughts' processes.

- The group theory and category theory application.

- Inverse problem solving on topological spaces, etc.

The development of AGI requires an interdisciplinary synthesis of different approaches, methods, and technologies. The synergy of these components of AGI has to overcome the nonfinite complexity of cognitive semantics. These effects imply the modeling beyond the traditional formalized mechanisms and models of thinking.

E.g., in the large paper [29] devoted to building machines that think like people note the importance of drawing a distinction between AI that purports to emulate or draw inspiration from human cognition and AI that does not. This paper focuses on the former that looks like AGI. The latter undoubtedly is a perfectly reasonable and useful approach to developing AI algorithms for real practice: avoiding cognitive inspiration and claims of neural plausibility. But for emergency requiring rapid collective decisions, this is clearly not enough.

The key aspects of AGI are creating cognitive semantics that can be embraced only by indirect approaches. One of these approaches is quantum semantics.

\section{Quantum Semantics}

The description of the disaster situation is usually infinitedimensional. In such cases, the mechanisms of creating quantum semantic interpretations for computer models can find practical application. The quantum principle of complementarity helps to expand and deepen the semantic interpretation of computer models. Applying the special quantum operators to sets of messages created during virtual conversations between participants $[8,9]$.

The uncaused nature of feelings, emotions, or free will force studying the quantum semantics approach [30,31]. The special quantum operator can help to embrace these unconscious components in decision-making processes [8].

A special place in the AGI approach is occupied by quantummechanical effects, such as superposition, complementarity, and non-locality effects [30]. For more complete and detailed coverage of the quantum and relativistic aspects of the cognitive semantics, an attempt can comprehensively study the thought processes using the group theory, which investigate sets with operations on them. The elements of spatial sets will then be various entities: events, ideal and virtual objects, complex numbers, vectors with their scalar products. Each event will be associated with a point in space, considered a mathematical object, different from others. Thus, 
both the Fourier transforms, and transformations over the 4dimensional Minkowski event space elements can be covered. The points of this space can have a non-numerical character, the coordinates of which serve as one of the many ways to specify these points. It helps to create cognitive semantics. The quantum cognitive semantics allow raising the power of AI substantially, e.g.:

- The quantum particle is represented in infinite-dimensional space - it may look like a symbolic representation of the spiritual phenomenon.

- The quantum particle is both a particle and a wave and gets another type in another place - it can reflect that a word's meaning depends on the context.

- Attempts to detect a wave of a quantum particle lead to the disappearance of the interference effect- it looks like the inability to logically describe cognitive semantics.

- The particle's behavior is described with the complex numbers - it can be used for representing the "shadow" components of the cognitive semantics.

- A quantum particle cannot have zero values of its parameters and occurs in a jump-like manner-it looks like the Eureka effect [16].

- The non-locality effect ensures the dependence of the quantum particle's behavior on other particles for which the behavior is not defined-it is associated with the interdependence of natural phenomena, etc.

The quantum semantics features increase the power of AI, making forecasting and solving processes more reliable. The way of realizing the possibilities of quantum semantics consists of emulating quantum operators on a common computer. For example, the paper [8] demonstrates using the Hadamard quantum operator, which interprets the quantum superposition function on a set of words with their frequencies (frequency histograms) in documents. The quantum operator changes the frequency histograms of words from the set of documents, making the coverage of these sets more complete. It also ensures the mapping of different sets of documents and words closed (in mathematical mean) that is the prerequisite for ensuring correctness of the inverse problems solving process (see sections 7, 8, and [4]).

\section{The Convergent Approach}

The collectives' decision-making processes have to be purposeful and sustainable in emergencies. The participants generate different ideas based on incoming information and make quick assessments of the various solutions' alternatives and suggestions. Creating ideas and making assessments include many interactions between participants using different units of information. The process may be influenced by false or latent information. This influence can greatly reduce the efficiency of group decision-making. This process may be divergent and has to be transformed into convergent (purposeful and sustainable).

Different types of operational objectives during group decision-making are suggested. Let's call them, for example, usual (traditional) and unusual (best) goals. Different values between these extreme values of the types of goals can be placed on the scale.

Usual objectives can be prescribed by automatically extrapolating retrospective statistics by traditional AI methods based on previous experience or retrospective information. The expert system, big data analysis, deep learning, ontology, etc. can be used for this aim. Most of the evaluations of those systems of goal setting are based on pattern recognition methods on retrospective data analysis.

For achieving the unusual, high-quality goals that cannot be achieved with traditional decision-support systems (DSS), the convergent approach was suggested [4, 8]. It has the opposite (inverse) character concerning DSS and AI. To ensure the efficiency of creating group high-level advice (decision-making), it is necessary to create special necessary structural conditions for support purposeful and sustainable development of the decisionmaking process. The convergent approach is based on the inverse problem-solving method on topological spaces and cognitive modeling. The provisions, algorithms, and programs to accelerate the decision-making process in emergencies with these methods were developed. The difference between the direct and inverse approaches is illustrated in Figure 3.

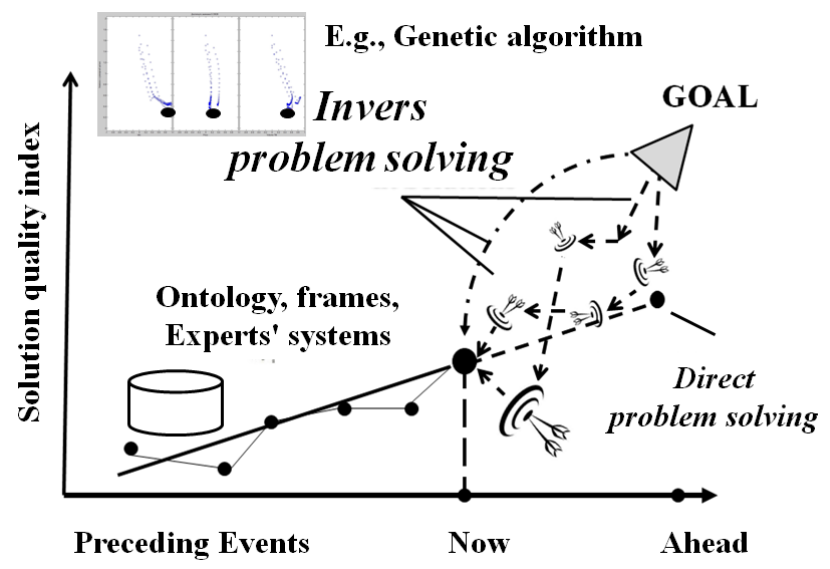

Figure 3: The difference between two approaches of decision-making

If participants of an emergency meeting try to use both these two different approaches (direct and inverse), they apply AI and AGI technologies. For inverse problem-solving, the Tychonoff theorem [32] on topological spaces was applied to ensure the meeting's stable purposefulness.

It was confirmed that the semantic interpretation of cognitive models in the decision-making process and the methods of inverse problems solving increase the purposefulness of the actions of the leader of a group of people (various services, rescue teams, spontaneous groups of people, etc.) and, therefore, the speed and quality of decision-making in emergencies.

The convergent approach provides the necessary conditions for the sustainable convergence of collective decision-making processes to ill-defined goals. To ensure these necessary conditions, category theory and the topology theory were used, and the concept of a "convergent monad" (CM) was introduced for which the classical monad was improved with the axioms of $\mathrm{CM}$ $\aleph[33]$ : 
- $\quad$ D: Top $\rightarrow$ Top; the number of points in the system of sets Top (topology) is infinite, and the graph of maps is closed (the complement of an open set).

- $\quad \aleph$ is a topological space where its open covers have nonempty finite subcover (The Compact space);

- $\quad$ Any two points of $\aleph$ (in the topological sense) have disjointed neighborhoods (The Hausdorff space).

These axioms follow from the statement: if $X$ and $Y$ are Hausdorff topological spaces and $X$ is a compact space, and the graph A is closed, these are necessary (but not sufficient) conditions for ensuring convergence of the inverse problem solving [34]. The convergent methodology allows dividing the problem into some parts and then assembling them into a holistic decision.

The following sets and an inverse operator were selected for reflecting the search of the solution in an emergency: the space of goals $Y$ (the exact goal $\mathrm{y}_{0}$ and the set of inaccurate goals $\left\{y_{\delta}\right\}$ ); the space of participants and means $X$ for achieving $\mathrm{y}_{0}$; the operator $A$ : $X \rightarrow Y$, and the reverse to it. The domain of definition of the operator A can be narrowed down to some subset of $X$, and the set of decisions can be narrowed down to a subset of $Y$ (Figure 4).

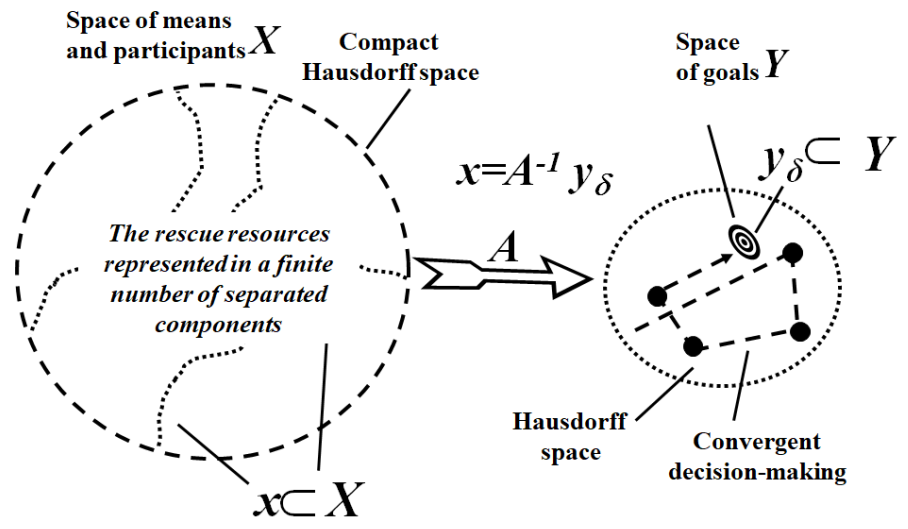

It is shown that a countable set and an infinite set of real numbers are clearly not suitable for identifying components and describing the space of solutions. This result allows supplementing the structure of the data using the requirements that were formed earlier [4]. For example, for increasing the convergence of a decision-making process, it is required that the number of parameters (factors) that characterize the problem situation should be finite. In this way, these factors and their semantics have to cover the description and representation of the problem and have to be separated, or the border could be created between every two parameters. Considering these requirements, a network brainstorming method based on a genetic algorithm was developed for inverse problem solving [35]. It can be used in emergency conditions for convergent group generation of ideas and formation of an agreed action plan.

Thus, for ensuring the stability and purposefulness of decisionmaking in emergencies, the components of the goals, rescue means, and ways (algorithm) of the achievement of the goals should be highlighted and structured especially. The emphasis should be focused on the possibility of accelerating the adoption of group decisions in networks and mobile environments and an interdepartmental system of distributed SC. The collective decision-making process involves the networked expertise approach, cognitive modeling, and mathematical apparatus for inverse problems solving $[5,7,8]$.

It was also shown [4] that to ensure the necessary conditions for a stable and purposeful synthesis of effective solutions in a problem situation; it is also advisable to distinguish the components: the whole, order, and chaos. To ensure the desired stability of decision-making processes, these components and their rate of change must be in a certain ratio (Figure 5).

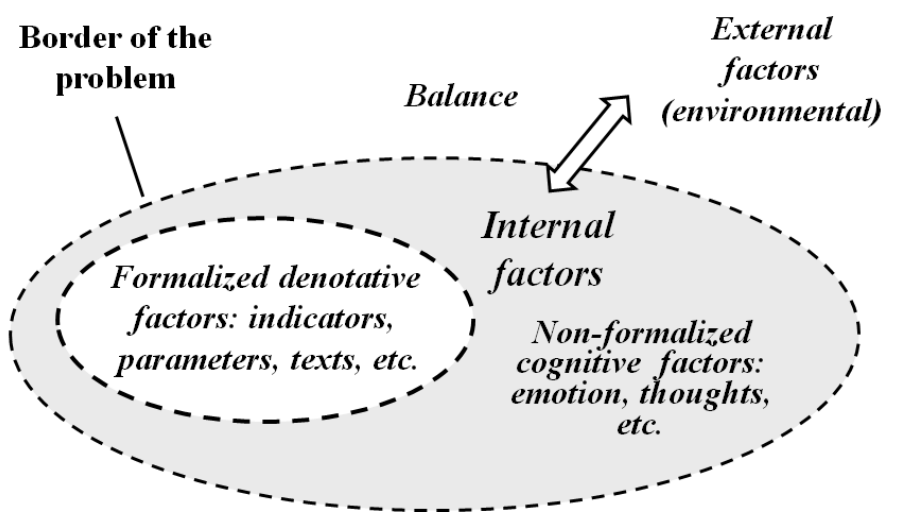

Figure 5: Chaotic component of ensuring the desired stability

The convergent approach offers a list of rules for structuring the information that is created during emergency meetings, as follows:

- The goals have to be constructed as a 3-level tree with main, external and internal goals;

- The goals must be ordered by importance with, for example, the analytic hierarchy method [36];

- Cognitive and denotative semantic interpretations of the model's factors, and their connections must be separated;

- The set of decisions must be represented by a finite and observable set of factors, etc.

Such recommendations help create conditions that ensure the purposefulness and sustainability of the decision-making process in an emergency. As shown by practical testing of the proposed approach's use with the creation of appropriate conditions, the duration of holding collective strategic meetings is reduced by $4-5$ times compared to cases when these conditions are not met. However, for emergencies, the acceleration of group decisionmaking should be significantly greater.

The participants and engaged network experts must have the possibility of putting qualitative information into the process of decision-making in an interactive way by using the cognitive modeling method $[4,5,35]$.

\section{Networked Expertise and Cognitive Modeling}

Different members can participate in disaster meetings to manage an emergency, and some remote experts can be invited [5]. On the base of convergent technology, networked expertise 
procedures can be ordered, as shown in Figure 6. This order accelerates the decision-making processes by speeding up getting agreement between the emergency meeting participants regarding goals and ways of action. The SC can centralize monitoring of the situation and ensure the coherence of actions of various teams and organizations.

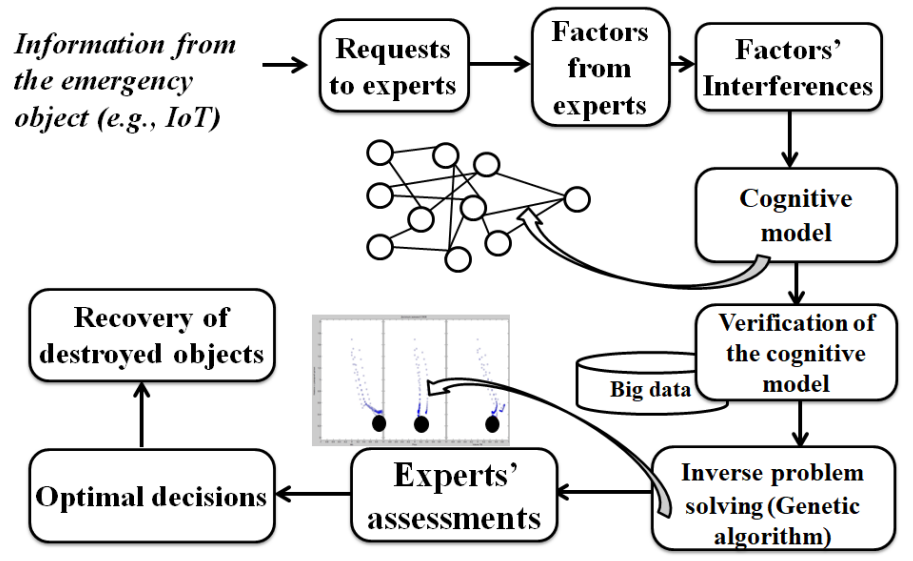

Figure 6: The order of the networked expertise and decision-making

The main steps of the networked expertise are as follows:

- Make the requests to the experts.

- Describe the situation shortly; formulate goals of improving the situation.

- Suggest some conceptual factors that characterize the opportunities to improve the situation.

- Define factors' interferences and create the cognitive model.

- Assess and explore the project of the cognitive model in remote SC.

- Solve the inverse problem with a genetic algorithm for getting the optimal decisions.

As a result of the network experts meeting, the final strategy of activity is formulated. The most difficult part of a group expert procedure is cognitive modeling. For simplifying and speeding up the process of cognitive modeling, an array of reference cognitive models, methods of Big Data analysis, and Deep learning should be used.

In a transport emergency, which claims fast decision-making and effective planning, the traditional manual creation of formalized computer models is impossible because it may take a lot of time. The problems have the characteristics of randomness, chaos, quantization, uncertainty, and non-causality. In these conditions, the participants must apply intuition, experience, and experts' judgments.

For such problems, the approach with the automation of cognitive modeling could be applied [37,38]. The cognitive modeling method is used when non-metric (qualitative) concepts are more important than metric (quantitative) ones. It ensures factors and relations between factors that characterize the problem. It may be represented in a matrix or a directed graph, where nodes represent factors, and arrows represent relations. The process helps to understand the structure of the problem and improves the understanding between participants.

The retrospective experience can help to create the cognitive model automatically. The creation of a cognitive model could be accelerated by using Big Data analysis. It helps to verify early prepared cognitive models and supply ones with denotative semantics in a formalized way. For this, the cognitive model's factors and their connections have to be mapped into the relevant subset of Big Data.

The deep learning of the neural network method was carried out for automating cognitive model creation and verification. The features of this process are as follows:

- Check the adequacy of the cognitive model to the problem situation.

- Ensure the availability of relevant subsets of Big Data.

- Use standard software for training the neural network.

An approach with a replacement of direct access to Big Data by addressing a deep neural network (DNN) was proposed to construct the denotative cognitive model's semantics. This replacement is that access to Big Data in an emergency cannot always be provided. The tool for permanent training of the DNN on retrospective examples for using in a concrete situation is worked out.

The expediency of using DNN was shown on the model with LSTM (Long Short-Term Memory), convolutional, and recurrent [39]. The LSTM model is well suited for classifying, processing, and predicting time series cases where time lags separate events with uncertain duration. The LSTM module can store values for both short and long periods of time. It does not use the activation function inside its recurrent components. The stages of creating a DNN are:

- Downloading the clusters of relevant documents from available data sets.

- Tokenization, lemmatization, and filtering of documents' texts.

- Constructing the LSTM model for training.

- $\quad$ Training the LSTM model.

The process has to consider qualitative aspects of the situation, ensuring the denotative and cognitive semantic interpretation of models. Participants conduct cognitive modeling in the following order:

- Some of the first factors of the emergency are revealed for creating the cognitive model.

- Some of the first mutual influences between factors are evaluated.

- The project of the cognitive model is created automatically with using Big Data analysis or DNN.

- The result of automated cognitive model creation is estimated by a group's leader.

- Goals and control factors in the model are identified. 
- The project of the cognitive model is corrected by a group's leader.

- The direct and inverse problems on the cognitive model are solving.

- The decision is suggested.

Figure 7 illustrates the preliminary creation and verification of cognitive models and synthesizes ones during decision-making.

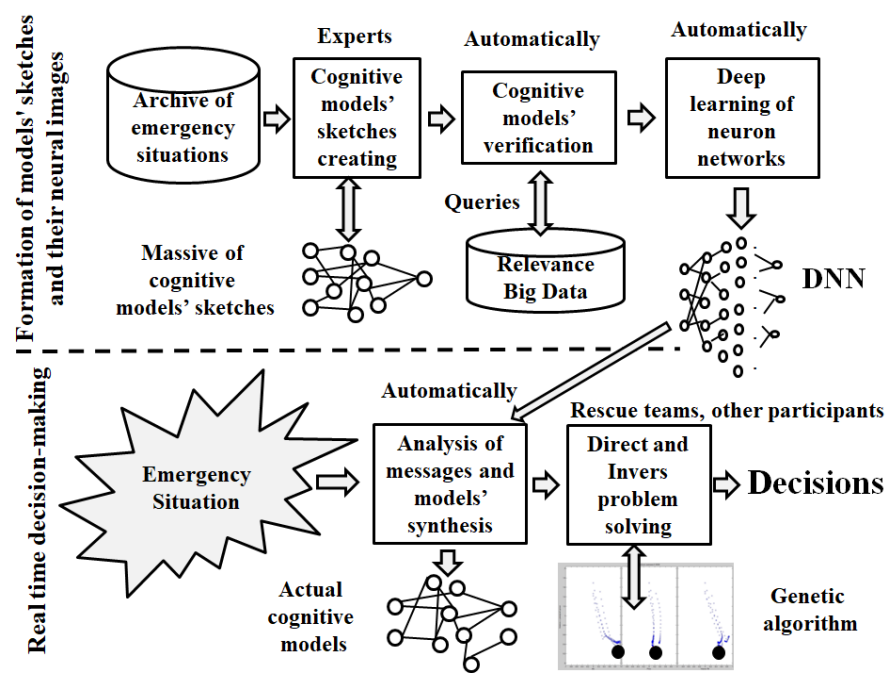

Figure 7: Cognitive model creation and verification

The testing was made on four different sample sizes of documents: 10,100,1000, and 4868. As the experiment showed, on the largest of these samples, the accuracy of determining the cognitive model factors reached $93 \%$. This result is so good that it allows raising the question to automate the cognitive model's construction in an emergency.

This group decision-making process in an emergency with the creation of a cognitive model may take only some minutes, and thereby it improves the process of decision-making and, as a result, increases the chances of saving human lives.

\section{Convergent Emergency Meeting in Practice}

The suggested convergent approach with cognitive modeling was exploited in the distributed SC for speeding up strategic conversations and emergency meetings. The convergent approach helps to reduce the duration of such meetings resolutely. The collective strategic meeting duration was reduced from four days to one day or even four hours.

But convergent distributed meetings in an emergency with the quick agreement of the participants on the emergency place and heads of departments of the authorities and experts still remain the subject for further implementation in practice.

As a result of applying the convergent approach, many drafts of the strategies for the socio-economic development of the cities, regions, and industries were created. During the strategic meeting (conversation), the group of 30-40 participants carries out brainstorming sessions, conducts a strategic analysis, creates a cognitive model, solves a direct and an inverse problem, and works out an optimal strategic solution. The main participants of the conversation and strategic planning are the administration of the controlled object, enterprise management, and remote experts.

Everybody from administration and rescue groups has to know the convergent approach's rules to reduce the duration of the emergency meeting by conducting and structuring the information in a convergent way. Currently, the duration of a strategic conversation takes around 4-6 hours. During this time, every participant makes many decisions regarding the goals of actions. It may coincide or not with the global decision of his group. The behaviors of various participants are different; changing the situation brings chaos to participants' minds.

For example, in [40], it is shown that the strategic meeting for creating the cognitive model of the large megapolis tourism development took 4 hours due to using the convergent methodology. For this, 35 brainstorming were run simultaneously. From the beginning, the goal tree was made. Then the SWOTanalysis (strengths, weaknesses, opportunities, and threats) helps to identify about 70 factors of the situation, which can influence the megapolis tourism development. After that, these factors were convoluted into the substantial 15 factors, and the weights of their interconnections were evaluated, and as a result, the cognitive model was made. Then the Big Data analysis helped to verify this model. Then the direct and inverse problems were solved on the cognitive model and an optimal scenario found.

Such a practice can be used in emergencies. In this case, the process of networked decision-making will cover casualties, groups of people, different rescue teams, administration's representatives, headquarters, experts, and groups' leaders. The problem is to find an agreement among all participants very quickly about aims and ways of action.

The convergent approach helps to synchronize the participants' behaviors. But the above-mentioned 4 hours that were spent during the strategic conversation for getting a consensus is an excessively long period of time, which is unacceptable in an emergency, and the decision-making process has to be substantially accelerated.

To further develop a convergent approach for speeding up decision-making in an emergency, appropriate research must be carried out in a classic and non-classical way [28]. Some of these studies are still debatable.

\section{Discussion}

The most complex and debatable issue that is the subject for further research is the construction of AI logical models' cognitive semantics. This construction is non-formalizable and cannot be described with the programmer's logic. Currently, studies are being conducted in the field of building quantum semantics and optical computing.

The decision-making process with cognitive modeling can be accelerated by using a quantum semantic approach. Quantum semantics make the models' interpretations more holistic. They help consider the phenomenon that every word of the texts of documents interferes with an infinite number of the hidden (shadow) words. It makes the possibility to create as much as possible complex virtual objects. On the other side, attempts to represent the cognitive semantics of words with only a discrete 
(digital) computer and logic give zero intersection ones with shadow words [38].

Creating cognitive semantics cannot be exactly reproduced by mathematical logic and discrete computers. They reduce the possibility of the cognitive semantic interpretation of the cognitive model and cannot consider the non-formalized processes of human thinking, emotions, thoughts, etc. For these aims, it will be useful to consider the opportunity of quantum entanglement (nonlocality) effects [30]. The Maxwell and Schrödinger equations were theoretically researched for compensating the cognitive semantics gaps. In this context, the potential of optical calculations using lasers, deflectors, and multidimensional rewritable holographic storage devices was theoretically investigated. It could be useful for creating cognitive semantics. [38].

Perhaps the unified mathematical approach may be the group theory, which can cover the convergence of collective decisionmaking processes, the formation of cognitive semantics using the quantum field theory, and relativistic theory. Apparently, this may require exploiting the transformations' operators in the Lorentz group, where fixed points represent the events, and the intervals between events (points) are preserved, or it may be useful to apply the Poincare group, where the fixation of points is not required.

Such an approach will place the symbolic AI model with its denotative (formalizable) semantics and, most importantly, cognitive (unformalizable) semantics in some single space, and then use unified operators to implement transformations of events of various nature. In this case, events can be attributed to different phenomena, described as by logic, as by quantum electrodynamics' laws and the special theory of relativity. If cognitive semantics allows a quantum interpretation with its representation in the infinite Hilbert space and a relativistic interpretation, with its representation in terms of the transformation of smooth manifolds (for example, to study fields' anomalies), then the application of group theory can be fruitful.

At the same time, the adequacy of the approach to the interpretation of cognitive semantics using group theory is a subject of lengthy verification, especially regarding its application to the possibility of joint research, primarily, of events that interpret these semantics at the subatomic level and the theory of relativity level.

Thus, the important discussion issue to substantially speed up decision-making during the rescue operation in transport emergency based on $\mathrm{AI}$ is constructing cognitive semantics of AI models, involving the mechanisms of subatomic and relativistic levels. Some ideas in this way were suggested in this paper.

\section{Conclusions}

To accelerate the processes of decision-making in transport emergency (air, rail, car, etc.), it is advisable to structure all generated information in a special way: making 3-level arranging the goals, dividing rescue means on finite numbers of components, automatic creating cognitive models with neural network deep learning, and applying an inverse problem-solving method by genetic algorithm on the cognitive model for finding the best decision. For this, the author's convergent approach was proposed that considers the non-formalized cognitive semantics of AI logical models and ensures the sustainability and purposefulness of decision-making processes.

During cognitive modeling and decision-making, different impacts on the same factors can give successful or destructive results. E.g., the order and strength of influence on the factors are critical for getting the desired results of modeling. The successful impact on the situation can be determined comparatively accurately with a genetic algorithm applied to the cognitive model.

The traditional logical approaches and deep learning methods in AI cannot cover cognitive semantics holistically. This semantics requires to be enriched by new approaches, such as quantum and relativistic theories, networked experts procedures, which have to be convergently organized, and different means like situational awareness tools and situational centers systems.

Thirty-five years of practical experience in applying the convergent approach has shown the possibility of drastically reducing collective situational analysis duration and building optimistic strategies. However, the application of a convergent approach for transport emergency requires its further development.

\section{Conflict of Interest}

The author declares no conflict of interest.

\section{Acknowledgment}

Supported by the Russian Foundation for Basic Research, grant No 18-29-03086 "Methods for identifying the needs of economic sectors in digital platforms and end-to-end technologies based on the analysis of large amounts of texts."

\section{References}

[1] M.R. Endsley, "Situation awareness in aviation systems," In: Garland, D.J., Wise, J.A., Hopkin, V.D., eds., Handbook of aviation human factors. Lawrence Erlbaum Associates, Mahwah, NJ, USA, 257-276, 1999.

[2] P.M. Salmon, G.H. Walker, N.A. Stanton, "Pilot error versus sociotechnical systems failure: a distributed situation awareness analysis of Air France 447," Theor Issues Ergon Sci, 17(1):64-79, 2016, doi: 10.1080/1463922X.2015.1106618.

[3] P. Illankoon, P. Tretten, U. Kumar, "Modelling human cognition of abnormal machine behavior," Hum-Intell Syst Integr, 1:3-26, 2019, https://doi.org/10.1007/s42454-019-00002-x.

[4] A.N. Raikov, "Convergent cognitype for speeding-up the strategic conversation,” IFAC Proc, 41(2):8103-8108, 2008, doi: 10.3182/200807065-KR-1001.01368.

[5] D. Gubanov, N. Korgin, D. Novikov, A. Raikov, E-expertise: Modern collective intelligence, Springer, 2014, https://doi.org/10.1007/978-3-31906770-4.

[6] A. Raikov, "Health care trust space based on collective artificial intelligence and blockchain technologies," J eHealth Technol Appl, 16(1):37-42, 2018.

[7] A. Raikov, "Accelerating technology for self-organising networked democracy," $\quad$ Futures, 103:17-26, 2018 , https://doi.org/10.1016/j.futures.2018.03.015.

[8] A. Raikov, "Convergent networked decision-making using group insights," Complex \& Intell Syst, 1(1): 57-68, December 2015, https://doi.org/10.1007/s40747-016-0005-9.

[9] A.N. Raikov, "Holistic discourse in the network cognitive modeling," J. Math Syst Sci, 3(10):519-530, 2013.

[10] B.B. Frey, J.H. Lohmeier, S.W. Lee, N. Tollefson, "Measuring collaboration among grant partners," Amer J Eval, 27(3):383-392, 2006, https://doi.org/10.1177/1098214006290356.

[11] G. Griffin, A. Rajabifard, and D. Williams, "The Victorian emergency management continuum and the benefits of spatial enablement," In: Spatially enabling government, industry and citizens (Global Geospatial Conference 2012), GSDI, 2012.

[12] M.R. Endsley, "Direct measurement of situation awareness: Validity and use of SAGAT," In: Endsley, M.R., Garland, D.J., eds., Situation awareness 
analysis and measurement, Lawrence Elbaum Associates, Mahwah, NJ, 131157, 2000. https://doi.org/10.4324/9781315087924-9.

[13] J.A. Crowder, J.N. Carbone, "Collaborative shared awareness: Human-AI collaboration," In: International conference on information and knowledge engineering, WorldComp, Athens, 1-6, 2014.

[14] D. Kahneman, "A psychological point of view: Violations of rational rules as diagnostic of mental processes," Behav Brain Sci 23:681-683, 2000, https://doi.org/10.1017/S0140525X00403432.

[15] A.A. Hadi, A.W. Mohamed, and K.M. Jambi, "LSHADE-SPA memetic framework for solving large-scale optimization problems," Complex Intell Syst. 5, 25-40, 2019. https://doi.org/10.1007/s40747-018-0086-8

[16] D. Perkins, The Eureka effect. The art and logic of breakthrough thinking, W.W. Norton \& Company, New York, London, 2000.

[17] K.R. Hammond, Principles of organization in intuitive and analytical cognition (report 231). Center for Research on Judgement and Policy. University of Colorado, Boulder, CO, 1981.

[18] M.R. Endsley, "Situation awareness: Progress and directions," In: Banbury, S., Tremblay, S., eds., A cognitive approach to situation awareness: Theory, measurement and application, Ashgate, Aldershot, 317-341, 2004.

[19] D.D. Heikoop, J.C.F. de Winter, B. van Arem, N.A. Stanton, "Psychological constructs in driving automation: A consensus model and critical comment on construct proliferation," Theor Issues Ergon Sci 17(3):284-303, 2004. https://doi.org/10.1080/1463922X.2015.1101507.

[20] H. Ibbara, R. Barbulescu, "Identity as narrative: Prevalence, effectiveness, and consequences of narrative identity work in macro work role transitions," Acad Manag Rev, 35(1):135-154, 2010. https://doi.org/10.5465/amr.35.1.zok135.

[21] C. Bolstad, M.R. Endsley, A.M. Costello, C.D. Howell, "Evaluation of computer-based situation awareness training for general aviation pilots," Int J Aviat Psychol, 20:269-294, 2010.

[22] N.A. Stanton, P. Salmon, G. Walker, D. Jenkins, "Is situation awareness all in the mind?," Theor Issues Ergon Sci 11(1-2):29-40, 2010. https://doi.org/10.1080/14639220903009938.

[23] I. Kotseruba, J.K. Tsotsos, "40years of Cognitive Architectures: Core Cognitive Abilities and Practical Applications," Artif Intell Rev, 53(1):7-94, 2020. https://doi.org/10.1007/s10462-018-9646-y.

[24] R.M. Pirsig, Zen and the art of motorcycle maintenance. William Morrow and Company, New York, 1974.

[25] R. Fjelland, "Why general artificial intelligence will not be realized," Humanit Soc Sci Commun 7(10), 2020. https://doi.org/10.1057/s41599-020-0494-4.

[26] N. Ilyin, G. Malinetsky, K. Kolin, A. Zatsarinny, et al., "Distributed situational centres system of cutting edge development," In: 10th international conference management of large-scale system development (MLSD), 2017. https://doi.org/10.1109/MLSD.2017.8109638.

[27] T.-J. Huang, "Imitating the brain with neurocomputer a 'new' way towards artificial general intelligence," Int J Autom Comput, 14(5):520-531, 2017. https://doi.org/10.1007/s11633-017-1082-y.

[28] A. Raikov, "Post-Non-Classical Artificial Intelligence and its Pioneer Practical Application.” IFAC-PapersOnLine. 52(25):343-348, 2019. https://doi.org/10.1016/j.ifacol.2019.12.547.

[29] B.M. Lake, T.D. Ullman, J.B. Tenenbaum, and S.J. Gershman, "Building machines that learn and think like people," Behavioral and Brain Sciences, Published online by Cambridge University Press, 40:1-70 e253, 2017. https://doi.org/10.1017/S0140525X16001837.

[30] H. Atmanspacher, "Quantum approaches to brain and mind: An overview with representative examples," in: Schneider, S., Velmans, M., eds., The Blackwell companion to consciousness, John Wiley, Chichester, 298-313, 2017. https://doi.org/10.1002/9781119132363.ch21.

[31] A. Dalela, Quantum meaning: A semantic interpretation of quantum theory. Shabda Press, 2012.

[32] C.K. Wong, "Covering properties of fuzzy topological spaces," J. Math Anal Appl, 43:697-704, 1973. https://doi.org/10.1016/0022-247X(73)90285-0.

[33] A.N. Raikov, A.N. Ermakov, and A.A. Merkulov, "Assessments of the economic sectors needs in digital technologies," Lobachevskii J Math, 40(11):1837-1847, 2019. https://doi.org/10.1134/S1995080219110246

[34] V.K. Ivanov, "Incorrect problems in topological spaces," Siberian Math J, 10(5):785-791, 1969. https://doi.org/10.1007/BF00971654.

[35] A.N. Raikov, S.A. Panfilov, "Convergent decision support system with genetic algorithms and cognitive simulation," IFAC Proc, 46(9):1108-1113, 2013. https://doi.org/10.3182/20130619-3-RU-3018.00404.

[36] T. L. Saaty, The analytic hierarchy process, University of Pittsburg, 1988.

[37] A.N. Raikov, Z. Avdeeva, and A. Ermakov, "Big data refining on the base of cognitive modeling," IFAC PapersOnLine, 49(32):147-152, 2016. https://doi.org/10.1016/j.ifacol.2016.12.205.

[38] A. Raikov, "Cognitive modelling quality rising by applying quantum and optical semantic approaches," IFAC PapersOnLine, 51(30):492-497, 2018. https://doi.org/10.1016/j.ifacol.2018.11.309.

[39] J. Goodfellow, Y. Bengio, A. Courville, Deep learning, The MIT Press, Cambrige, Massachusets, London, England, 2017.

www.astesj.com
[40] A. Raikov, "Megapolis Tourism Development Strategic Planning with Cognitive Modelling Support,” In: Yang XS., Sherratt S., Dey N., Joshi A (eds), Fourth International Congress on Information and Communication Technology (London). Advances in Intelligent Systems and Computing, vol. 1041. Springer, Singapore, 2020. https://doi.org/10.1007/978-981-15-0637612. 